\title{
A Novel WNK1-PDGFRB Fusion Gene in Myeloid Neoplasm With Eosinophilia: A Case Report With a Literature Review
}

\section{Tiantian Wang}

The Affiliated People's Hospital of Ningbo University

Junjie Cao

The Affiliated People's Hospital of Ningbo University

Qingqing Lin

The Affiliated People's Hospital of Ningbo University

Xuhui Liu

The Affiliated People's Hospital of Ningbo University

Man Wang

The First Affiliated Hospital of Soosuzhou University

Renzhi Pei

The Affiliated People's Hospital of Ningbo University

Ying Lu ( $\square$ luying0820@hotmail.com )

The Affiliated People's Hospital of Ningbo University

\section{Case Report}

Keywords: WNK1-PDGFRB, Myeloid neoplasms, RNA-seq, Imatinib, PDGFRB

Posted Date: February 23rd, 2022

DOI: https://doi.org/10.21203/rs.3.rs-1374875/v1

License: (9) This work is licensed under a Creative Commons Attribution 4.0 International License. Read Full License 


\section{Abstract}

Platelet-derived growth factor receptor beta (PDGFRB) rearrangements play an important role in the pathogenesis of eosinophilia-associated myeloid/lymphoid neoplasms. Here, a novel WNK1-PDGFRB fusion gene has been identified in myeloid neoplasm with eosinophilia by RNA-sequencing. This is the first study that reported on the WNK1-PDGFRB fusion gene expression in myeloid neoplasm with eosinophilia. In addition, the WNK1-PDGFRB fusion retained the entire kinase domain of PDGFRB and response to imatinib at low concentration.

\section{Background}

In 2008, the World Health Organization (WHO) defined eosinophilia with PDGHRA, PDGFRB, or FGFR1 gene mutation as a new myeloid neoplasm. In 2016, the WHO released a report stating that myeloid/lymphoid neoplasm with eosinophilia are usually associated with PDGHRA, PDGFRB, FGFR1, and PCM1-JAK2 fusion gene [1, 2]. PDGFRB fusion gene was first described in a patient with features consistent with chronic myelomonocytic leukemia (CMML) in 1994, Since that time, over 40 fusion partners have been described. Myeloid neoplasm with eosinophilia accompanied by PDGFRB gene rearrangement is more common in men, where the ETV6-PDGFRB gene is the most prevalent one. Imatinib mesylate is considered a valid therapeutic option for this condition $[3,4]$.

This study describes a new WNK1-PDGFRB fusion gene in a patient with myeloid neoplasm.

\section{Case Report}

A 56-year-old man was admitted to the Affiliated People's Hospital of Ningbo University in September 2021. He had a one-year history of paroxysmal dizziness and an abnormal hemogram. Physical examination showed splenomegaly. Laboratory tests revealed leukocytosis $\left(18.8 \times 10^{9} / \mathrm{L}\right)$, with increased eosinophil levels $\left(3.95 \times 10^{9} / \mathrm{L}\right)$. A remarkable proportion of eosinophils were found in the bone marrow, including eosinophilic myelocyte (6\%), eosinophilic metamylocyte (6\%), eosinophilic stab granulocyte $(5 \%)$, and eosinophilic segmented granulocyte ( $8 \%)$ (Fig. 1A). The neutrophil alkaline phosphatase score was 3. Flow cytometry immunophenotyping wasCD34+, CD16+, CD11b+, CD14+.

Biochemical index, total IgE, parasite check, thyroid function, and autoantibodies were all within normal levels. Karyotype analysis showed 46, XY, t(5;12)(q33;p13)[11]/46, XY[9]. The BCR-ABL fusion gene was negative, and ETV6-PDGFRA, ETV6-PDGFRB, FIP1L1-PDGFRA, FIP1L1-RARA, KIF5B-PDGFRA, STRNPDGFRA, TELetv6-PDGFRB, and PCM1-JAK2 were not detected. Sixty-six blood tumor gene mutations were negative, including JAK2V617F, MPL 515, CALR, and c-kit mutations(Patient's DNA was extracted using the Aidlab Whole Blood Genome DNA Extraction Kit. The NovaSeq platform was used for sequencing. Used the Burrows-Wheeler Alignment (BWA) tool MEM algorithm to align the readings with the human genome reference sequence (GRCh38.p13). Identification of SNPs and INDELs, processing of the generated BAM files using GACK 3.8, Samtools, VarScan.v2.3.9 software to generate VCF files. All 
mutations were annotated using ANNOVAR software. Then, we performed fluorescence in situ hybridization (FISH) on BCR-ABL, PDGFRA, PDGFRB, and FGFR1. Preliminary results showed that $89 \%$ of cells carried PDGFRB gene rearrangement at the 5q32-33 (Figure B). Patient's RNA was extracted from BM cell by Trizol methods according to the manufacturer's protocol (Invitrogen, Waltham, MA) for RNAsequencing (RNA-seq) in September 2021. RNA quality and concentration were estimated by Nano Drop ND-2000 (Thermo Fisher Scientific, Waltham, MA). Paired-end reads were generated from the complementary DNA (cDNA) libraries using an Illumina Next Seq 550 instrument (Illumina, San Diego, CA). Then we used star-fusion software to analyze the RNA-seq raw data (Supplementary Material). Standard settings were applied for all three tools and reads were aligned to the Genome Reference Consortium Human Build 37 (GRCh37). A new WNK1-PDFRB fusion gene was discovered identified (Fig. 1C). To confirm the fusion, Digital - polymerase chain reaction (Digital-PCR) was performed: Primers and probes corresponding to WNK1-23F primer: AGACGACGACCCACTAAAAGC, PDGFRB-11R2: primer: ATGATAAGGGAGATGATGGTGAGC and WNK1-23P primer: AGTCGAAGCAGTTCCT were adopted to prepare the dPCR system (Rui Xun Biological Technology Co, LTD). The chips were imaged by QuantStudio 3D Digital PCR Instrument (Thermo Fisher, \#4489084) and analyzed using the QuantStudio 3D Analysis Suite Cloud Software.WNK1-PDFRB Fusion /ABL1 quantitative ratio is $164.797 \%$ (Fig. 1D).

Imatinib was daily used at the dose of $200 \mathrm{mg}$ by October 2021 . Two months after oral treatment, the laboratory tests revealed that WBC was $2.7 \times 10^{9} / \mathrm{L}$ with $1.6 \%$ eosinophil in the peripheral blood. The patient obtained sustained complete hematologic remission.

\section{Discussion}

The PDGFRB gene is located in the $5 q 22$, which encodes platelet-derived growth factor cell surface receptors. So far, more than 40 PDGFRB associated fusion genes have been found [5]. T (5;12) (q31-q33; $\mathrm{p} 12)$ is a common chromosomal aberration, and ETV 6 is the most common fusion gene [6]. However, $t$ $(5 ; 12)$ (q31-q33; p12) do not cause ETV6-PDGFRB gene mutation [7]. Due to the heterogeneity and falsenegative results of cytogenetics and FISH, recognizing the PDGFRB associated gene is challenging [810]. Chen et a/ reported a new CSNK2A1-PDGFRB fusion gene in a 37-year-old man with eosinophilia, while other PDGFRB fusion gene was negative by RT-PCR and FISH were all negative [11]. In this study, we used the target RNA sequencing test and found that the WNK1 at Chromosome 12p13 fused with PDGFRB at Chromosome 5q32 resulted in a WNK1-PDGFRB (Fig. 1C,1D).

With-No-Lysine Kinase 1 (WNK1) belongs to the WNK family, which is on the 12p13 chromosome, is widely expressed in tissues such as the brain, spinal cord, kidney, heart, skeletal muscle, and testis. It has a pivotal role in tumor and cardiovascular disease by regulating cell proliferation and death [12]. But it is rare reported in in myeloid neoplasms. WNK1 is activated by downstream receptor tyrosine kinase through the MAPK pathway in various tumors [13]. In the wNK1-PDGFRB fusion protein, WNK1 retains the oxidative - stress - responsive kinase $1 \mathrm{C}$ - terminal domain, and PDGFRB retains the fusion protein kinase region. Therefore, we speculated that WNK1 could be activated by downstream PDGFRB fusion gene protein kinase region through MAPK pathway, resulting in eosinophil proliferation. 
Imatinib is the first therapy choice for patients with PDGFRB rearrangement. Yet, so far, no consensus has been reached on initial and maintenance dose $[6,14]$. It is considered that a low dose of 100 to $200 \mathrm{mg}$ of imatinib can lead to good outcomes. Chen et al reported that the patient acquired sustained molecular complete remission for 2 years until imatinib at the dose of $200 \mathrm{mg}$ [9]. Another report showed that the patient received complete remission by an initial $400 \mathrm{mg}$ dose until the blood cell count normalized and with a maintenance dose of $100 \mathrm{mg}$ for more 7 months. The patient obtained sustained complete hematologic remission and normalization of spleen[14]. Our patient successfully responded to the $200 \mathrm{mg}$ daily imatinib treatment. Yet, long-term efficacy and dose adjustment need to be further evaluated.

\section{Conclusion}

To the best of our knowledge, this is the first study that reported on the WNK1-PDGFRB fusion gene expression in myeloid neoplasm with eosinophilia.

\section{Declarations}

\section{Consent}

Written informed consent was obtained from the patient for publication of this case report and any accompanying images.

\section{Authors' contributions}

T.T.W and Y.L. were the principal investigator who took primary responsibility for this study. T.T.W wrote the manuscript. R.Z.P., X.H. L, and J.J.C studies and commented on the manuscript. Q.Q.L and M.W performed experiments and participated in clinical data management. All authors read and approved the final manuscript.

\section{Funding}

This research was supported by the Ningbo and Yinzhou District Social and Development project (20191JCGY020007), China.

\section{Declarations}

Ethics approved this study was conducted in accordance with the principles of the Declaration of Helsinki and approved by the ethics committee of the institution. Informed consent was obtained from this patient.

\section{Conflict of interest}

The authors declared no competing interests. 
Availability of data and materials

Data sharing not applicable to this article as no datasets were generated or analyzed.

\section{Acknowledgments}

Thanks to Professor Chen Suning, Director Shen Hongjie of the First Affiliated Hospital of Soosuzhou University for their theoretical guidance and technical support.

\section{References}

1. Arber DA, Orazi A, Hasserjian R, et al $\varangle 2016 \rrbracket$. The 2016 revision to the World Health Organization classification of myeloid neoplasms and acute leukemia. Blood. 127(20):2391-2405. Blood, 2016. 128(3): 462-463.doi:10.1182/blood-2016-06-721662.

2. A Reiter and J Gotlib (2017). Myeloid neoplasms with eosinophilia. Blood. 129(6): 704714.doi:10.1182/blood-2016-10-695973.

3. M C Dispenza and B S Bochner\2018囚. Diagnosis and Novel Approaches to the Treatment of Hypereosinophilic Syndromes. Curr Hematol Malig Rep. 13(3): 191-201.doi:10.1007/s11899-0180448-8.

4. M Shekarabi, N Girard, J B Riviere, P Dion, M Houle, A Toulouse, et al $22008 \rrbracket$. Mutations in the nervous system--specific HSN2 exon of WNK1 cause hereditary sensory neuropathy type II. J Clin Invest. 118(7): 2496-505.doi:10.1172/JCl34088.

5. X Wu, H Cai, Y Qiu, J Li, D B Zhou, and X X Cao®2020区. ETV6-ACSL6 fusion gene in myeloid neoplasms: clinical spectrum, current practice, and outcomes. Orphanet J Rare Dis. 15(1): 192.doi:10.1186/s13023-020-01478-6.

6. D Di Giacomo, M Quintini, V Pierini, F Pellanera, R La Starza, P Gorello, et al邓2022】. Genomic and clinical findings in myeloid neoplasms with PDGFRB rearrangement. Ann Hematol. 101(2): 297307.doi:10.1007/s00277-021-04712-8.

7. P Erben, D Gosenca, M C Muller, J Reinhard, J Score, F Del Valle, et al. $\$ 2010 \otimes$ Screening for diverse PDGFRA or PDGFRB fusion genes is facilitated by generic quantitative reverse transcriptase polymerase chain reaction analysis. Haematologica. 95(5): 73844.doi:10.3324/haematol.2009.016345.

8. X Xu, Q Lu, Z Wang, P Cai, Z Zeng, L Zhang, et alष2021区. Identification of a Novel CSNK2A1-PDGFRB Fusion Gene in a Patient with Myeloid Neoplasm with Eosinophilia. Cancer Res Treat. 53(3): 889892.doi:10.4143/crt.2020.1272.

9. J Gotlib囚2017囚. World Health Organization-defined eosinophilic disorders: 2017 update on diagnosis, risk stratification, and management. Am J Hematol. 92(11): 1243-1259.doi:10.1002/ajh.24880.

10. A R Rodan and A Jenny $82017 \rrbracket$. WNK Kinases in Development and Disease. Curr Top Dev Biol, . 123: 1-47.doi:10.1016/bs.ctdb.2016.08.004. 
11. M Andrei, A Bandarchuk, C Abdelmalek, A Kundra, V Gotlieb, and J C Wang $₫ 2017$. PDGFRRearranged Myeloid Neoplasm with Marked Eosinophilia in a 37-Year-Old Man; And a Literature Review. Am J Case Rep. 18: 173-180.doi:10.12659/ajcr.900623.

12. N Von Bubnoff, S P Gorantla, S Thone, C Peschel, and J Duyster 2006 . The FIP1L1-PDGFRA T674I mutation can be inhibited by the tyrosine kinase inhibitor AMN107 (nilotinib). Blood. 107(12): 4970-1; author reply 4972.doi:10.1182/blood-2006-01-0285.

13. N M Butt, J Lambert, S Ali, P A Beer, N C Cross, A Duncombe, et al $82017 \rrbracket$. Guideline for the investigation and management of eosinophilia. Br J Haematol. 176(4): 553572.doi:10.1111/bjh.14488.

14. H Fang, G Tang, S Loghavi, P Greipp, W Wang, S Verstovsek, et al『2020冈. Systematic use of fluorescence in-situ hybridisation and clinicopathological features in the screening of PDGFRB rearrangements of patients with myeloid/lymphoid neoplasms. Histopathology. 76(7): 10421054.doi:10.1111/his.14097.

\section{Figures}


A

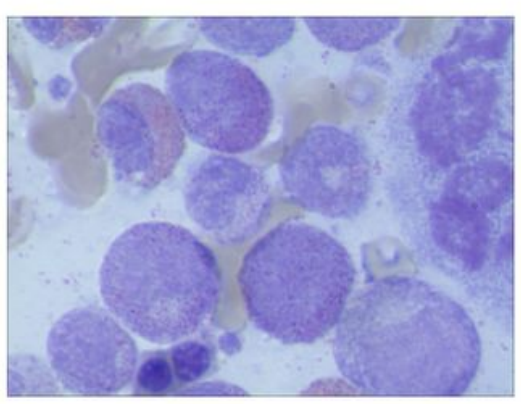

C

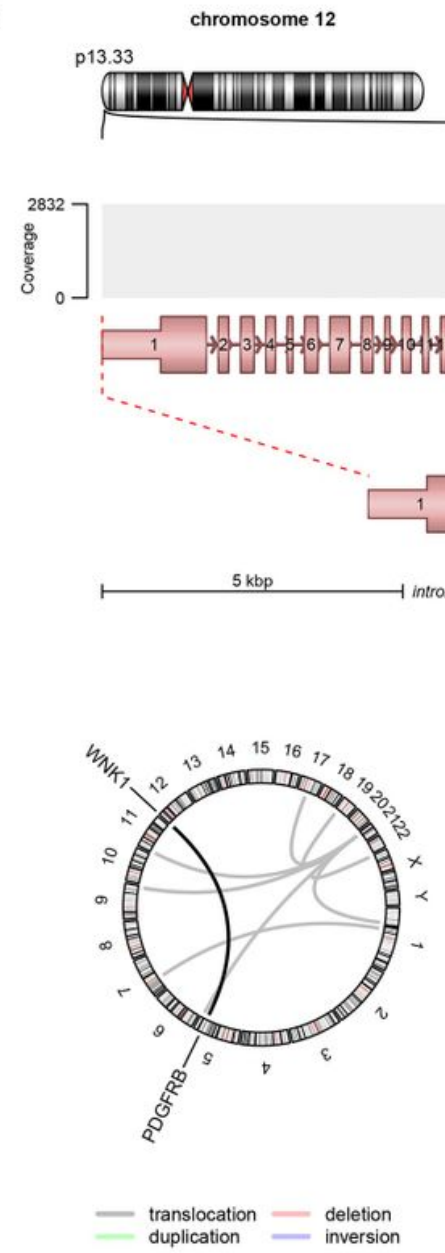

B

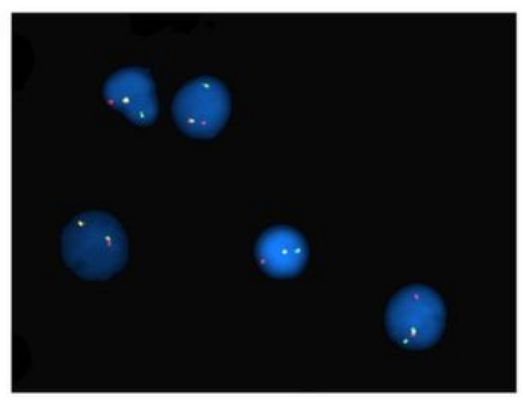

D

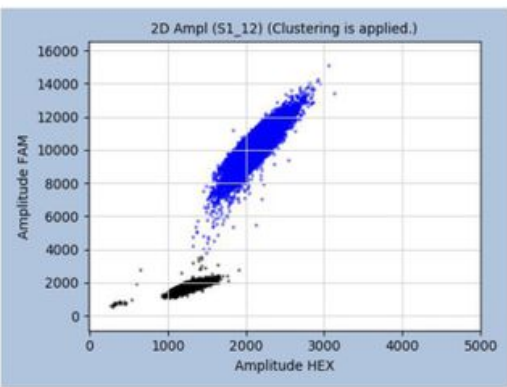

chromosome 5

q32

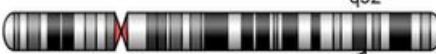

breakpoint
5:150126614

breakpoint

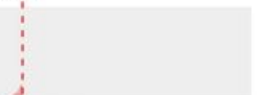

4

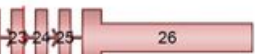

232425

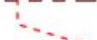

ENST00000535572.5

,...
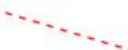

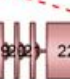

$22-25124$ और 4 (5)

TCCTTGGGGAATAAAAGCCCCCAGCTTTCAGCCTTGCCCTITAAGGTGGTGGTGATCTCAG

Mitut
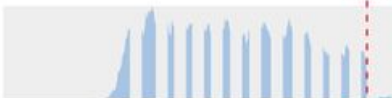

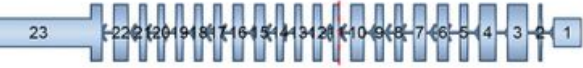

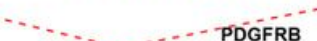

ENST00000261799

\section{Figure 1}

Identification of novel WNK1-PDGFRB fusions. (A)Bone marrow aspirate showing prominent eosinophilia ( $\times 100$ magnification): May-Grünwald-Giemsa staining showing eosinophilia in the diagnostic bone marrow aspirate. (B) The PDGFRB locus at $5 q 32-33$ in $89 \%$ of cells by FISH: nuc ish(5هPDGFRB $\times 2) \rrbracket(3 \rrbracket$ PDGFRB $\times 2) \rrbracket(5 区 P D G F R B$ sep $3 \llbracket P D G F R B \times 1)[356 / 400]$. (C) The fusion between chromosome 12 of the WNK11 gene (12: 897681) and chromosome 5 of the PDGFRB gene (5: 150126614). (D) WNK1-PDFRB Fusion / ABL1 quantitative ratio is $164.797 \%$ by using Digital-PCR.

\section{Supplementary Files}


This is a list of supplementary files associated with this preprint. Click to download.

- Dataavailability51FusionGeneReports.pdf

- DataavailabilityBloodTumorGeneMutationReport.pdf

- DataavailabilityChromosome.pdf

- DataavailabilityPCM1JAK2fusiongenereport.pdf

- DataavailabilityPCM1JAK2fusiongenereportd1.pdf

- DataavailabilityRNASeq.pdf

- DataavailabilityETV6PDGFRfusiongene.pdf

- DataavailabilityFISHreport.pdf

- DataavailabilityWNK1PDGFRBDigitalPCR.pdf 